\title{
Nonalcoholic Fatty Liver Diseases among Recently Diagnosed Patients with Diabetes Mellitus and Risk Factors
}

\author{
Amna S Butt ${ }^{1}$, Saeed Hamid ${ }^{2}$, Zishan Haider ${ }^{3}$, Fatima Sharif ${ }^{4}$, Mohammad Salih $^{5}$, Safia Awan ${ }^{6}$, Aatif A Khan ${ }^{7}$, Jaweed Akhter $^{8}$
}

\begin{abstract}
Aim: Prevalence of obesity and type 2 diabetes mellitus (T2DM), both of which represent are related to nonalcoholic fatty liver disease (NAFLD), is an increasing trend among Asian people. The study aimed to assess the prevalence of NAFLD in T2DM with their risk factors in the Southern part of Pakistan.

Materials and methods: A cross-sectional study was accomplished during 2008-2013 at The Aga Khan University Hospital, Karachi, Pakistan. Adult patients diagnosed with T2DM during last 6 months were enrolled in this study. NAFLD was identified using ultrasound of the liver. Clinical and biochemical relevant measurements were accomplished.

Results: Out of a total of 203 patients with T2DM, NAFLD was detected in 146 patients (71.9\%). Multivariate analysis revealed that NAFLD was significantly associated with dyslipidemia (OR $2.38,95 \% \mathrm{Cl} 1.06-5.34, p=0.035)$, higher LDL (OR 1.02, 95\% $\mathrm{Cl} 1.01-1.03, p=0.003), \mathrm{HbA} 1 \mathrm{C}$ (OR1.27, 95\% Cl 0.97-1.68, $p=0.045$ ) and diastolic blood pressure (OR 1.05, 95\% Cl 1.01-1.10, $p=0.009$ ). The highest odds of 10.8 for NAFLD ( $95 \% \mathrm{Cl} 4.9-24, p=0.001$ ) was found for the combination of hypertension, dyslipidemia, body mass index (BMI), waist circumference, lack of physical inactivity, triglycerides, lower HDL, LDL, HbA1c, and ALT (multiplicative analysis).

Conclusion: High incidence of NAFLD with the association of different lifestyle-related factors has been analyzed. It unmasks the need for screening for NAFLD in newly diagnosed DM patients in Pakistan with the assessment of parameters of risk factors.

Keywords: Diabetes mellitus, Nonalcoholic fatty liver disease, Risk factors, Prevalence.

Euroasian Journal of Hepato-Gastroenterology (2019): 10.5005/jp-journals-10018-1288
\end{abstract}

\section{INTRODUCTION}

$\mathrm{N}$ onalcoholic fatty liver disease (NAFLD) may range from simple steatosis to nonalcoholic steatohepatitis (NASH) and has an increasing trend in the world as well as in Asia., ${ }^{1,2}$ These pathological processes may progress to development of cirrhosis and/or hepatocellular carcinoma (HCC) and are also associated with several systemic disorders such as colorectal cancer, cardiovascular and metabolic conditions. ${ }^{3}$ Based upon limited data, it seems that about 5-20\% people in Asia may have NAFLD, but it varies considerably based on location, gender, race, and age. ${ }^{4}$ Also, an absence of symptom and health alarms in the majority of patients with NAFLD makes its diagnosis and management more complicated. ${ }^{2,5}$

The diagnosis of NAFLD can be made by assessing the history of alcohol intake, levels of liver enzymes (ALT, SGOT, GGT), evidence of hepatic steatosis on imaging, while more confirmatory diagnosis can be made by the presence of steatosis along inflammation or fibrosis on liver biopsy. ${ }^{6,7}$ Among noninvasive imaging modalities, ultrasonography has been found more sensitive and cost-effective then computerized tomography or MRI scan. ${ }^{2,5}$

Obesity, hypertension, and dyslipidemia have all been observed in NAFLD, but the most important metabolic disorder implicated in this condition is T2DM. ${ }^{8}$ Whether present in isolation or concurrently with metabolic syndrome, T2DM has been strongly associated with NAFLD because of the occurrence of insulin resistance. ${ }^{9}$ Furthermore, higher rates of overall mortality, mortality related to liver and cardiovascular diseases has been reported when T2DM and NAFLD were present concomitantly. ${ }^{10,11}$ These factors and co-factors are on a rising trend in Asian countries. ${ }^{12,13}$ This might be due to the difference in body composition of Asians from Caucasians in terms of proportion and distribution of body fat, hence making Asians genetically predisposed to the occurrence of insulin resistance in the absence of high grades of obesity. ${ }^{13}$
1,2,5 Section of Gastroenterology, Department of Medicine, Aga Khan
University Hospital, Karachi, Pakistan
${ }^{3}$ Department of Radiology, Aga Khan University Hospital, Karachi, Pakistan

${ }^{4}$ Aga Khan University, Karachi, Pakistan

${ }^{6,7}$ Department of Medicine, Aga Khan University Hospital, Karachi, Pakistan

${ }^{8}$ Section of Endocrinology, Department of Medicine, Aga Khan University Hospital, Karachi, Pakistan

Corresponding Author: Amna S Butt, Section of Gastroenterology, Department of Medicine, Aga Khan University Hospital, Karachi, Pakistan, Phone: 009221-34864508, e-mail: amna.subhan@aku.edu

How to cite this article: Butt AS, Hamid S, et al. Nonalcoholic fatty Liver Diseases Among Recently Diagnosed Patients With Diabetes Mellitus and Risk Factors. Euroasian J Hepatogastroenterol 2019;9(1):9-13.

Source of support: Supported by University Research Council, Aga Khan University, Karachi, Pakistan

Conflict of interest: None

However, only limited data is available from Pakistan in this regard where periodic screening and surveillance of diabetics is not a common practice in NAFLD. Hence, the majority of patients with NAFLD remain untreated. The lack of awareness regarding burden, associated conditions, and risk of potential complications of NAFLD will probably further increase the disease burden on the Pakistani population.

This study was aimed to assess the prevalence of NAFLD among Pakistani patients with newly diagnosed T2DM. We also targeted to determine the predicting factors associated with NAFLD. This will provide information about disease burden among diabetics and will

() The Author(s). 2019 Open Access This article is distributed under the terms of the Creative Commons Attribution 4.0 International License (https://creativecommons. org/licenses/by-nc/4.0/), which permits unrestricted use, distribution, and non-commercial reproduction in any medium, provided you give appropriate credit to the original author(s) and the source, provide a link to the Creative Commons license, and indicate if changes were made. The Creative Commons Public Domain Dedication waiver (http://creativecommons.org/publicdomain/zero/1.0/) applies to the data made available in this article, unless otherwise stated. 
also promote the concept of primary prevention and intervention at an early stage. Moreover, this may also provide a foundation for further interventional projects for the management of NAFLD to reduce NAFLD-related complications.

\section{Materials AND Methods}

\section{Recruitment of Participants}

The study represents a prospective, hospital-based cross-sectional study conducted from 2008-2013 at the Gastroenterology and Endocrinology outpatient clinics of The Aga Khan University Hospital, Karachi, Pakistan. Consecutive adult patients diagnosed to have T2DM during the last 6 months were enrolled after receiving informed consent. Those patients who refused to participate in the study, who had history of significant alcohol intake ( $>40$ $\mathrm{g}$ per week) or had prior chronic liver diseases, those who were taking hepatotoxic drugs like statins, thiazolidinediones (TZDs), tamoxifen, nucleoside reverse transcriptase inhibitors, protease inhibitors, had short bowel syndrome or were on total parenteral nutrition were excluded from this study. Information was collected regarding demographic characteristics, medications, presence of comorbidities and risk factors. All patients were examined physically to derive anthropometric measurements. Blood tests were done to measure laboratory parameters and ultrasound was done to assess the fatty liver disease.

Body weight and height were measured, and body mass index (BMI) was calculated. On the basis of World Health Organization (WHO) guidelines for the Asian population; subjects with a BMI between $23-25 \mathrm{~kg} / \mathrm{m}^{2}$ were considered overweight whereas those with a $\mathrm{BMI} \geq 25$ were labeled obese. ${ }^{14}$ The waist circumference and hip circumference were also measured; the waist-to-hip ratio was calculated. Blood pressure (BP) was also measured. DM was defined based on WHO criteria (fasting plasma glucose, $\geq 126 \mathrm{mg} / \mathrm{dL}$, or the two hours post-load plasma glucose $\geq 200 \mathrm{mg} / \mathrm{dL}$. ${ }^{15}$ The Joint National Committee (JNC) ${ }^{7}$ criteria were used to define hypertension (HTN). ${ }^{16}$ Metabolic syndrome was evaluated based on the criteria of the National Cholesterol Education Program Adult Treatment Panel III (NCEP, ATP III). ${ }^{17}$ According to ATP III, at least two of the following criteria should be fulfilled to define metabolic syndrome (BP $\geq 130 / 85$ $\mathrm{mm} \mathrm{Hg}$, high triglycerides ( $\mathrm{TG}>150 \mathrm{mg} / \mathrm{dL}$ ); low high-density lipoprotein ( $\mathrm{HDL}<40 \mathrm{mg} / \mathrm{dL}$ in men, $<50 \mathrm{mg} / \mathrm{dL}$ in women), or waist circumference as per Asian criteria for central obesity of $>90 \mathrm{~cm}$ (men) or $>80 \mathrm{~cm}$ (women). All patients underwent an assessment of fasting blood sugar (FBS), fasting lipid profile (FLP), alanine aminotransferase (ALT), and hemoglobin A1c (HbA1c). Other underlying liver diseases were excluded by checking hepatitis B (HBsAg) and hepatitis C (anti-HCV antibody), ceruloplasmin, antinuclear antibody (ANA), antismooth muscle antibody (ASMA) and antimitochondrial antibody (AMA).

A single experienced sonographer performed all ultrasounds who was blinded regarding other details of study participants. Presence of bright liver, increased hepatic echotexture than the renal echotexture, presence of vascular blurring and narrowing of hepatic veins were used to detect NAFLD on ultrasound. ${ }^{18,19}$ Moreover, presence of fine diffuse hyperechogenicity was considered as grade I (mild steatosis), moderate but diffuse hepatic hyperechogenicity, impaired visualization of intrahepatic vessels and diaphragm was considered grade II (moderate steatosis). ${ }^{19}$ While the diagnosis of grade III (severe steatosis) was made if there was marked hepatic hyperechogenicity, borders of intrahepatic vessels, and impaired visualization of diaphragm, and posterior portion of right lobe of liver. ${ }^{19}$

\section{Ethical Considerations}

The study received permission of the ethical review committee of AKUH (732-Med/ERC-07).

\section{Sample Size and Statistical Analysis}

Using the software EPI Info, ${ }^{20}$ we assessed the prevalence of NAFLD by assuming $20 \%$ of NAFLD in general population $5 \%, 21 \%$ and $48 \%$ in patients with DM. ${ }^{6}$ Taking a difference of $28 \%$ in prevalence, with $95 \%$ confidence level, a bond on error of \pm 2.2 and dropout rate of $10 \%$, the estimated sample size of 240 patients was required. Statistical package for social science (SPSS 19.0) was used to analyze the data. Mean \pm standard deviation was calculated for quantitative variables and proportions were calculated for categorical variables. Comparative analysis was done using student t-test or Pearson Chi-square or Fisher exact test whenever appropriate. To identify risk factors associated with NAFLD univariate and multiple logistic regression analysis was accomplished. To estimate the multiplicative effect for the combination of various risk factors binary logistic analysis was done. A $p$ value of less than 0.05 was considered statistically significant.

\section{Results}

A total of 500 patients with newly diagnosed T2DM were screened, out of which 230 patients were found eligible. Out of 230, 20 participants were excluded due to incomplete workup or lost to follow up and 7 were excluded for nonadherence to inclusion criteria, and thus 203 patients were available for final analysis. The clinical profiles of the patients have been given in Table 1. Most of them had high fasting and random blood sugars, higher HBA1c and lower HDL levels (Table 1). Overall NAFLD was found in 146 (71.9\%) cases and out of them, $71(48.6 \%), 63$ (43.15\%) and 12 (8.2\%) patients had Grade I, II and III NAFLD, respectively. Although liver biopsy was offered to confirm the diagnosis, only five patients accepted and underwent liver biopsy which revealed Grade II NAFLD in 4 cases and NASH in one case.

Furthermore, two groups of patients have compared i.e., patients without NAFLD (group A) vs. patients with NAFLD (group B). A significant proportion of patients without NAFLD were doing some physical activity like brisk walk or exercise as compared to group B $(p<0.0001)$. The prevalence of hypertension $(75.3 \%$ vs. $57.9 \%, p 0.01)$, dyslipidemia $(78.1 \%$ vs. $54.4 \%, p 0.002)$, higher systolic (133.99 \pm 14.4 vs. $125.28 \pm 18.19$ $\mathrm{mm} \mathrm{Hg}, p<0.0001)$ and diastolic BP (83.77 \pm 11.63 vs. $76.98 \pm 7.90$ $\mathrm{mm} \mathrm{Hg}, p<0.0001$ ) were found in group $B$ as compared to group A. Likewise, the patients having NAFLD were more obese $(85.6 \%$ vs. $59.6 \%, p<0.0001)$, had a higher waist circumference $(98.77 \pm$ 12.58 vs $91.67 \pm 16.28 \mathrm{~cm}, p 0.001)$ and higher hip circumference $(104.79 \pm 11.59$ vs. $95.98 \pm 15.09 \mathrm{~cm}, p<0.0001)$ as opposed to those without NAFLD. Moreover, significantly higher levels of FBS $(152.2 \pm 50.8$ vs. $133.7 \pm 44.7 \mathrm{mg} / \mathrm{dL}, p 0.01)$, RBS $(219.86 \pm 86.19$ vs. $186.70 \pm 63.16, p 0.009), \mathrm{HbA1C}(8.13 \pm 1.69$ vs. $7.27 \pm 1.29, p$ $0.001)$, triglycerides (200.3 $\pm 101.2 \mathrm{vs} .151 .4 \pm 75.3 \mathrm{mg} / \mathrm{dL}, 0.001)$, LDL $(117.3 \pm 35.0$ vs. $92.1 \pm 34.0 \mathrm{mg} / \mathrm{dL},<0.0001)$, and ALT $(28.9 \pm$ 19.1 vs. $21.9 \pm 13.5,0.01$ ) were found in patients having NAFLD than patients without NAFLD (Table 1). Overall 26 (12.8\%) study subjects had elevated ALT and the proportion of study subjects with higher ALT was higher in those who of Group B compared to group A ( $16.4 \%$ vs. $3.5 \%, p 0.01)$. 
Table 1: Demographic and clinicpathological characteristics of all patients at baseline and comparison of patients with and without NAFLD $(n=203)$

\begin{tabular}{|c|c|c|c|c|}
\hline & & & Comparison & \\
\hline & All patients $(n=203)$ & Without NAFLD $(n=57)$ (group A) & With NAFLD $(n=146)$ (group B) & $p$ value \\
\hline Age (years) & $52.49 \pm 9.10$ & $52.61 \pm 7.64$ & $52.44 \pm 9.63$ & 0.90 \\
\hline Gender & & & & \\
\hline Male & $104(51.2)$ & $31(54.4)$ & $73(50.0)$ & 0.64 \\
\hline Female & $99(48.8)$ & $26(45.6)$ & $73(50.0)$ & \\
\hline Physical activity & & & & \\
\hline Yes & $117(57.6)$ & $38(66.7)$ & $48(32.9)$ & $<0.0001$ \\
\hline No & $86(42.4)$ & $19(33.3)$ & $98(67.1)$ & \\
\hline HTN & & & & \\
\hline No & $60(29.6)$ & $24(42.1)$ & $36(24.7)$ & 0.01 \\
\hline Yes & $143(70.4)$ & $33(57.9)$ & $110(75.3)$ & \\
\hline Dyslipidemia & & & & \\
\hline No & $58(28.6)$ & $26(45.6)$ & $32(21.9)$ & 0.002 \\
\hline Yes & $145(71.4)$ & $31(54.4)$ & $114(78.1)$ & \\
\hline Systolic BP (mm Hg) & $131.55 \pm 16.00$ & $125.28 \pm 18.19$ & $133.99 \pm 14.4$ & $<0.0001$ \\
\hline Diastolic BP $(\mathrm{mm} \mathrm{Hg})$ & $81.87 \pm 11.12$ & $76.98 \pm 7.90$ & $83.77 \pm 11.63$ & $<0.0001$ \\
\hline Waist circumference $(\mathrm{cm})$ & $96.77 \pm 14.05$ & $91.67 \pm 16.28$ & $98.77 \pm 12.58$ & 0.001 \\
\hline Hip circumference (cm) & $102.32 \pm 13.24$ & $95.98 \pm 15.09$ & $104.79 \pm 11.59$ & $<0.0001$ \\
\hline Waist to hip ratio & $0.94 \pm 0.06$ & $0.95 \pm 0.05$ & $0.94 \pm 0.06$ & 0.27 \\
\hline BMI categories $\left(\mathrm{kg} / \mathrm{m}^{2}\right)$ & & & & \\
\hline $18-22.9$ & $13(6.4)$ & $5(8.8)$ & $8(5.5)$ & $<0.0001$ \\
\hline $23-24.9$ & $31(15.3)$ & $18(31.6)$ & $13(8.9)$ & \\
\hline$\geq 25$ & $159(78.3)$ & $34(59.6)$ & $125(85.6)$ & \\
\hline $\mathrm{FBS}(\mathrm{mg} / \mathrm{dL})$ & $147.08 \pm 49.79$ & $133.75 \pm 44.73$ & $152.28 \pm 50.84$ & 0.01 \\
\hline $\mathrm{RBS}(\mathrm{mg} / \mathrm{dL})$ & $210.55 \pm 81.62$ & $186.70 \pm 63.16$ & $219.86 \pm 86.19$ & 0.009 \\
\hline HBA1C & $7.88 \pm 1.63$ & $7.27 \pm 1.29$ & $8.13 \pm 1.69$ & 0.001 \\
\hline Total cholesterol (mg/dL) & $172.18 \pm 43.49$ & $159.81 \pm 43.16$ & $177.01 \pm 42.81$ & 0.01 \\
\hline Triglycerides (mg/dL) & $186.60 \pm 97.01$ & $151.42 \pm 75.31$ & $200.34 \pm 101.21$ & 0.001 \\
\hline $\mathrm{HDL}(\mathrm{mg} / \mathrm{dL})$ & $38.43 \pm 8.89$ & $42.09 \pm 7.96$ & $37.01 \pm 8.85$ & $<0.0001$ \\
\hline $\mathrm{LDL}(\mathrm{mg} / \mathrm{dL})$ & $110.26 \pm 36.50$ & $92.14 \pm 34.00$ & $117.33 \pm 35.07$ & $<0.0001$ \\
\hline $\begin{array}{l}\text { Alanine transaminase } \\
(\mathrm{IU} / \mathrm{L})\end{array}$ & $\begin{array}{l}27 \pm 18.01 \text { (range } \\
7-150)\end{array}$ & $21.95 \pm 13.59$ & $28.97 \pm 19.14$ & 0.01 \\
\hline ALT categories & & & & \\
\hline Normal & $177(87.2 \%)$ & $55(96.5)$ & $122(83.6)$ & 0.01 \\
\hline Elevated & $26(12.8 \%)$ & $2(3.5)$ & 24 (16.4) & \\
\hline
\end{tabular}

The data of univariate analysis has been shown in Table 2 and this data shows a relation between several lifestyle-related factors with NAFLD. By the way, physical activity and higher HDL were found to be protective factors against NAFLD on univariate analysis. The data of multivariate analysis has been cited in Table 3 that indicate the importance of dyslipidemia, higher LDL HbA1c, and diastolic blood pressures were factors significantly associated with NAFLD. Physical activity and higher were protective factors significantly associated with NAFLD.

Multiplicative effect for the combination of risk factors using binary logistics and its association with NAFLD is presented in
Figure 1. The odds of NAFLD with two risk factors ranged from 2.2 (physical inactivity and HTN or physical inactivity and TG) to 3.7 (waist circumference and TG). A rising trend in odds of having NAFLD was observed with an increasing number of risk factors.

\section{Discussion}

About $72 \%$ of our patients with newly diagnosed DM also had NAFLD based on ultrasound criteria. It seems that a high prevalence of NAFLD in patients having newly diagnosed DM has been reported by this article; however, a conclusive comment is yet to be given as the incidence of NAFLD may vary considerably 
Nonalcoholic Fatty Liver Diseases among Recently Diagnosed Patients with Diabetes Mellitus and Risk Factors

Table 2: Univariate analysis for factors associated with NAFLD in patients with newly diagnosed type $2 \mathrm{DM}$

\begin{tabular}{|c|c|c|}
\hline & $\begin{array}{l}\text { OR }(95 \% \text { confidence } \\
\text { interval) }\end{array}$ & $p$ value \\
\hline Age (years) & $0.99(0.96-1.03)$ & 0.99 \\
\hline \multicolumn{3}{|l|}{ Gender } \\
\hline Female & 1.0 & \\
\hline Male & $1.19(0.64-2.20)$ & 0.57 \\
\hline \multicolumn{3}{|l|}{ Physical activity } \\
\hline No & 1.0 & \\
\hline Yes & $0.24(2.13-7.82)$ & $<0.0001$ \\
\hline \multicolumn{3}{|l|}{ HTN } \\
\hline No & 1.0 & \\
\hline Yes & $2.22(1.16-4.24)$ & 0.01 \\
\hline \multicolumn{3}{|l|}{ Dyslipidemia } \\
\hline No & 1.0 & \\
\hline Yes & $2.98(1.55-5.73)$ & 0.001 \\
\hline Systolic BP (mm Hg) & $1.03(1.01-1.06)$ & 0.001 \\
\hline Diastolic BP(mm Hg) & $1.06(1.03-1.10)$ & $<0.0001$ \\
\hline Waist circumference $(\mathrm{cm})$ & $1.04(1.01-1.06)$ & 0.002 \\
\hline Hip circumference (cm) & $1.06(1.03-1.09)$ & $<0.0001$ \\
\hline Waist to hip ratio & $0.06(0.00-8.95)$ & 0.27 \\
\hline \multicolumn{3}{|l|}{ BMI categories $\left(\mathrm{kg} / \mathrm{m}^{2}\right)$} \\
\hline $18-22.9$ & 1.0 & \\
\hline $23-24.9$ & $0.45(0.12-1.69)$ & 0.24 \\
\hline$\geq 25$ & $2.29(0.71-7.47)$ & 0.16 \\
\hline FBS (mg/dL) & $1.01(1.00-1.01)$ & 0.02 \\
\hline RBS (mg/dL) & $1.01(1.00-1.01)$ & 0.01 \\
\hline HBA1C & $1.51(1.18-1.94)$ & 0.001 \\
\hline Total cholesterol (mg/dL) & $1.01(1.00-1.01)$ & 0.01 \\
\hline Triglycerides (mg/dL) & $1.01(1.00-1.01)$ & 0.001 \\
\hline $\mathrm{HDL}(\mathrm{mg} / \mathrm{dL})$ & $0.93(0.90-0.97)$ & $<0.0001$ \\
\hline LDL (mg/dL) & $1.02(1.01-1.03)$ & $<0.0001$ \\
\hline $\begin{array}{l}\text { Alanine transaminase } \\
\text { (IU/L) }\end{array}$ & $1.03(1.00-1.06)$ & 0.01 \\
\hline \multicolumn{3}{|l|}{ ALT categories } \\
\hline Normal & 1.0 & \\
\hline Elevated & $5.41(1.23-23.69)$ & 0.02 \\
\hline
\end{tabular}

depending on various factor. ${ }^{10,21,22}$ The overall prevalence of NAFLD in South-East Asia varies from $9 \%$ to $45 \%$ in general population and $6-62 \%$ in patients with DM. ${ }^{23}$ Among South Asian countries even higher prevalence of NAFLD has been reported in patients with DM (India 30-90\% and Sri Lanka 55\%). ${ }^{24,25}$ However, this is the first study from Pakistan which was conducted in a systematic way to address this issue.

The present study used ultrasound to diagnose NAFLD which is considered a suitable first-line screening method for NAFLD for a larger group of patients where the acceptance rate for liver biopsy will be low. It is cost effective and widely available diagnostic
Table 3: Multivariate analysis for risk factors associated with NAFLD in patients with newly diagnosed type $2 \mathrm{DM}$

\begin{tabular}{lll}
\hline & $\begin{array}{l}\text { OR (95\% confidence } \\
\text { Interval) }\end{array}$ & p value \\
\hline Physical activity & $0.23(0.10-0.50)$ & $<0.0001$ \\
$\mathrm{LDL}(\mathrm{mg} / \mathrm{dL})$ & $1.02(1.01-1.03)$ & 0.003 \\
$\mathrm{HDL}(\mathrm{mg} / \mathrm{dL})$ & $0.92(0.87-0.96)$ & $<0.0001$ \\
Dyslipidemia & $2.38(1.06-5.34)$ & 0.035 \\
Diastolic BP $(\mathrm{mm} \mathrm{Hg})$ & $1.05(1.01-1.10)$ & 0.009 \\
HbA1c & $1.27(0.97-1.68)$ & 0.045 \\
\hline
\end{tabular}

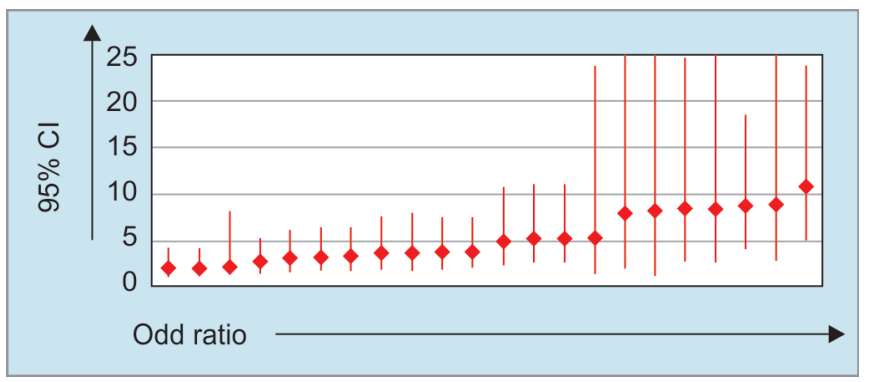

Fig. 1: Odds of NAFLD against combination of various risk factors

modality commonly used in clinical practice, however, its yield is lower in obese patients and in those having CLD. ${ }^{26,27}$ Although liver biopsy could provide more concrete information about NAFLD, and this could not be accomplished in this study due to its invasive nature. It is used mostly when there is a need to stage fibrosis, monitor disease prognosis and success of therapy. ${ }^{2,28}$

The proportion of patients with hypertension, obesity, higher waist, and hip circumference was significantly higher among those who had NAFLD. Similar findings have been reported by Kalra et al. from India. ${ }^{29,30}$ Likewise, higher levels of fasting and random blood sugars, LDL and HbA1c were found with NAFLD in our study. This was consistent with the association of factors included in metabolic syndrome and DM. ${ }^{18,31}$ Since, HbA1c is a known marker of uncontrolled DM the link between $\mathrm{HbA1c}$ and NAFLD could be explained well.

Several biochemical parameters were elevated in DM patients with NAFLD (levels of ALT). ALT is considered as a surrogate/ marker for hepatic inflammation and indirectly reflects NASH, if elevated. ${ }^{31,32}$ In our study, overall $26(12.8 \%)$ study subjects had elevated ALT and the proportion of study subjects with higher ALT was higher in those who had NAFLD (16.4\% vs. 3.5\%, p 0.01) as compared to group $A$. These findings were consistent with data reported from India. ${ }^{33}$

On multivariate analysis, several blood parameters were significantly associated with NAFLD in our study. Similar association including the association of NAFLD with diastolic BP has been reported in a recent study from Sri Lanka. ${ }^{34}$ Daily exercise had a protective factor in concordance with previous studies. ${ }^{35,36}$

An important finding in our study was related to combined effects of various risk factors which have not been evaluated in most of earlier studies. The odds of NAFLD with two risk factors ranged from 2.2 (physical inactivity and HTN or physical inactivity and TG) to 3.7 (waist circumference and TG). This is an alarming fact necessitating the need for early management and primary prevention for potential risk factors and NAFLD. 
Large scale, multicenter or population-based studies would be required to provide a solid background of the present analysis. Randomized control trials which assess the effect of lifestyle modifications including dietary changes and exercise on glycemic control and severity of NAFLD would be useful in devising new management targets in this patient population. Because most of the diabetic patients are being treated by endocrinologist or physicians, and it has been observed that they screen diabetic patients for diabetic retinopathy, neuropathy, and nephropathy but not for NAFLD. Our study has highlighted the high burden of NAFLD in patients with DM. So, it would be important to increase awareness of physicians and internists regarding the association of NAFLD and DM. Early detection, proper counseling of patients will help to prevent the long term complications associated with NAFLD and will also help to reduce the burden of disease too.

\section{Authors contributions}

Butt and Hamid contributed to study conception and design; Data collection, analysis, and manuscript was written by Butt, Haider performed all ultrasounds, Sharif, Awan and Khan assisted in data collection and writing; further review and finalization of manuscript was done by Butt, Hamid, Sharif.

\section{References}

1. Younossi ZM, Stepanova M, et al. Changes in the prevalence of the most common causes of chronic liver diseases in the United States from 1988 to 2008. Clin Gastroenterol Hepatol 2011;9(6):524-530. e1.

2. Chalasani N, Younossi Z, et al. The diagnosis and management of non alcoholic fatty liver disease: Practice Guideline by the American Association for the Study of Liver Diseases, American College of Gastroenterology, and the American Gastroenterological Association. Hepatology 2012;55(6):2005-2023.

3. Baffy Gr, Brunt EM, et al. Hepatocellular carcinoma in non-alcoholic fatty liver disease: an emerging menace. J Hepatol. 2012;56(6):13841391.

4. Ashtari S, Pourhoseingholi MA, et al. Non-alcohol fatty liver disease in Asia: Prevention and planning. World J Hepatol 2015;7(13):1788.

5. Fan JG, Zhu J, et al. Prevalence of and risk factors for fatty liver in a general population of Shanghai, China J Hepatol 2005;43(3):508-514.

6. ClarkJM,DiehIAM.Nonalcoholic fatty liver disease:an underrecognized cause of cryptogenic cirrhosis. JAMA 2003;289(22):3000-3004.

7. Dasarathy S, Dasarathy J, et al. Validity of real time ultrasound in the diagnosis of hepatic steatosis: a prospective study. J Hepatol 2009;51(6):1061-1067.

8. Hassan K, Bhalla V, et al. Nonalcoholic fatty liver disease: A comprehensive review of a growing epidemic. World J Gastroenterol 2014;20(34):12082.

9. Bhat G, Baba CS, et al. Insulin resistance and metabolic syndrome in nonobese Indian patients with non-alcoholic fatty liver disease. Trop Gastroenterol 2013;34(1):18-24.

10. Targher G, Bertolini L, et al. Prevalence of nonalcoholic fatty liver disease and its association with cardiovascular disease among type 2 diabetic patients. Diabetes Care 2007;30(5):1212-1218.

11. Younossi ZM, Gramlich T, et al. Nonalcoholic fatty liver disease in patients with type 2 diabetes. Clin Gastroenterol Hepatol 2004;2(3):262-265.

12. Shaw JE, Sicree RA, et al. Global estimates of the prevalence of diabetes for 2010 and 2030. Diabetes Res Clin Pract 87(1):4-14.

13. Yoon $\mathrm{K}-\mathrm{H}$, Lee $\mathrm{J}-\mathrm{H}$, et al. Epidemic obesity and type 2 diabetes in Asia. The Lancet 2006;368(9548):1681-1688.
14. Appropriate body-mass index for Asian populations and its implications for policy and intervention strategies. Lancet (London, England) 2004;363(9403):157-163.

15. Alberti KG, Zimmet PZ. Definition, diagnosis and classification of diabetes mellitus and its complications. Part 1: diagnosis and classification of diabetes mellitus provisional report of a WHO consultation. Diabet Med 1998;15(7):539-553.

16. Chobanian AV, Bakris GL, et al. The Seventh Report of the Joint National Committee on Prevention, Detection, Evaluation, and Treatment of High Blood Pressure: the JNC 7 report. JAMA 2003;289(19):2560-2572.

17. Huang PL. A comprehensive definition for metabolic syndrome. Dis Model Mech 2009;2(5-6):231-237.

18. Fan JG, Saibara T, et al. What are the risk factors and settings for nonalcoholic fatty liver disease in Asia-Pacific? J Gastroenterol Hepatol 2007;22(6):794-800.

19. Joseph AEA, Saverymuttu SH, et al. Comparison of liver histology with ultrasonography in assessing diffuse parenchymal liver disease. Clin Radiol 1991;43(1):26-31.

20. EPI Info: a word processing database and statistics program for public health [computer program]. Version 6. Atlanta GCfDCaP, 1995.

21. Angulo P. Nonalcoholic fatty liver disease. N Engl J Med 2002; 346(16): 1221-1231.

22. Kasturiratne $A$, Weerasinghe $S$, et al. Influence of non alcoholic fatty liver disease on the development of diabetes mellitus. J Gastroenterol Hepatol 2013;28(1):142-147.

23. Farrell GC, Wong VW, et al. NAFLD in Asia--as common and important as in the West. Nat Rev Gastroenterol Hepatol 2013;10(5):307-318.

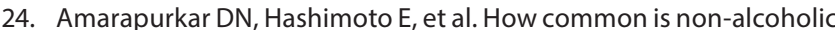
fatty liver disease in the Asia-Pacific region and are there local differences? J Gastroenterol Hepatol 2007;22(6):788-793.

25. Farrell GC. Non-alcoholic steatohepatitis: what is it, and why is it important in the Asia-Pacific region? J Gastroenterol Hepatol 2003;18(2):124-138.

26. de Moura Almeida A, Cotrim HP, et al. Fatty liver disease in severe obese patients: diagnostic value of abdominal ultrasound. World J Gastroenterol 2008;14(9):1415.

27. Perez NE, Siddiqui $F A$, et al. Ultrasound diagnosis of fatty liver in patients with chronic liver disease: a retrospective observational study. J Clin Gastroenterol 2007;41(6):624-629.

28. Nascimbeni F, Pais R, et al. From NAFLD in clinical practice to answers from guidelines. J Hepatol. 2013;59(4):859-871.

29. Kalra S, Vithalani M, et al. Study of prevalence of nonalcoholic fatty liver disease (NAFLD) in type 2 diabetes patients in India (SPRINT). J Assoc Physicians India 2013;61(7):448-453.

30. Fan J-G, Zhu J, et al. Prevalence of and risk factors for fatty liver in a general population of Shanghai, China. J Hepatol 2005;43(3):508-514.

31. Aller R, Izaola $\mathrm{O}$, et al. Predictive factors of non-alcoholic steatohepatitis: relationship with metabolic syndrome. Nutricion Hospitalaria 2015;31(6):2496-2502.

32. Fraser $A$, Harris R, etal. Alanineaminotransferase, $\hat{l}^{3}$-glutamyltransferase, and incident diabetes The British Women's Heart and Health Study and meta-analysis. Diabetes Care 2009;32(4):741-750.

33. Krishnan AVJ. Prevalence of nonalcoholic fatty liver disease and its biochemical predictors in patients with type-2 diabetic mellitus. E\&C Hepatology 2011;7(3-4):7-10.

34. Dassanayake AS, Kasturiratne A, et al. Prevalence and risk factors for non-alcoholic fatty liver disease among adults in an urban Sri Lankan population. J Gastroenterol Hepatol 2009;24(7):1284-1288.

35. Ueno $T$, Sugawara $H$, et al. Therapeutic effects of restricted diet and exercise in obese patients with fatty liver. J Hepatol 1997;27(1): 103-107.

36. Suzuki A, Lindor K, et al. Effect of changes on body weight and lifestyle in nonalcoholic fatty liver disease. J Hepatol 2005;43(6):1060-1066. 